\title{
Audição de crianças com fissura labiopalatina e baixo peso: estudo comparativo
}

\section{Hearing in children with cleft lip and palate and low weight: comparative study}

Alyne Michelle de Freitas Lima', José Roberto Pereira Lauris², Mariza Ribeiro Feniman³.

1) Graduação. Acadêmica do Curso de Fonoaudiologia da Faculdade de Odontologia de Bauru - USP.

2) Livre Docência. Professor Associado do Departamento de Odontopedia, Ortodontia e Saúde Coletiva da Faculdade de Odontologia de Bauru - USP

3) Pós-Doutorado em Audiology. Professora Titular do Departamento de Fonoaudiologia-FOB-USP.

Instituição: Faculdade de Odontologia de Bauru - Universidade de São Paulo/FOB-USP. Bauru / SP - Brasil

Endereço para correspondência: Mariza Ribeiro Feniman - Alameda Octavio Pinheiro Brisolla, 9-75 - Vila Universitária - Bauru / SP - Brasil - CEP: $17012-901$ - Telefone: (+55 14) 3235-8000 - E-mail: feniman@usp.br

Artigo recebido em 22 de Março de 2011. Artigo aprovado em 30 de Junho de 2011

\section{RESUMO}

Introdução: A presença de baixo peso ao nascer (BP), assim como a fissura labiopalatina (FLP), acabam por tornar as crianças mais propensas às alterações auditivas.

Objetivo: verificar e comparar a audição de crianças com fissura labiopalatina com e sem baixo peso ao nascimento na avaliação audiológica convencional.

Método: Foi realizado estudo retrospectivo e comparativo no que se refere ao gênero, peso ao nascimento, presença/ausência de FLP e ao resultado da audiometria e imitanciometria. Três grupos de crianças foram constituídos. G1 com 23 FLP e BP, G2 com 25 FLP e sem BP e G3 com 25 sem FLP e sem BP.

Resultados: Não foi encontrada diferença estatística significante entre os grupos na comparação do peso e gênero, bem como quanto, ao gênero e orelhas direita e esquerda, em relação ao tipo da curva timpanométrica, presença de perda auditiva, tipo de perda auditiva e grau da perda auditiva.

Conclusão: Um maior comprometimento da audição, evidenciado pela presença de perda auditiva condutiva de grau leve a moderada foi encontrado nas crianças com fissura labiopalatina (G1 e G2), independente da presença de baixo peso ao nascimento, quando comparada as sem este tipo de malformação craniofacial.

Palavras-chave: criança, fissura palatina, peso ao nascer.

\section{SUMMARY}

Introduction: The presence of low weight at birth (LW), as well as cleft lip and palate (CLP), end up making children more prone to auditory alterations.

Objective: Verify and compare the hearing of children with cleft lip and palate with and without low weight at birth in the conventional audiological evaluation.

Method: A retrospective and comparative study was made concerning gender, weight at birth, presence/absence of CLP and the result of audiometry and immittance measurements. Three groups of children were formed. G1 with 23 CLP and LW, G2 with 25 CLP but not LW and G3 with 25 children with neither CLP or LW.

Results: There wasn't found any statistically significant differences between the groups in none of the following comparisons: the weight and gender, gender and right/left ear, in relation to the kind of tympanometric curve, presence of hearing loss, type of hearing loss and degree of the hearing loss.

Conclusion: A greater auditory impairment, evidenced by the presence of light to moderate degree of conductive hearing loss, was found in children with cleft lip and palate (G1 and G2), regardless of the presence of low weight at birth, when compared to those without this kind of malformation.

Keywords: child, cleft palate, weight at birth. 


\section{INTRODUÇÃO}

Estudos apontam como responsáveis pelo aumento da sobrevivência de crianças prematuras, neonatos com baixo peso e de recém-nascidos com outros comprometimentos severos, os avanços tecnológicos e científicos. Em contrapartida, surgem casos em que fatores como o baixo peso ao nascer, podem causar aumento da morbidade neonatal e atraso no desenvolvimento global (1).

Obaixo peso ao nascimento está entre os principais fatores neonatais de alto risco. Estudiosos (2) classificaram o baixo peso ao nascimento entre 1500 a 2500g; peso normal entre 2500 a $4000 \mathrm{~g}$ e, macrossômico o peso superior a $4000 \mathrm{~g}$.

Existem intercorrências pré, peri e pós-natais que podem causar deficiência auditiva e estas intercorrências caracterizam os indicadores de risco (3). Vários autores relacionam essas intercorrências ao aparecimento de alterações sensoriais, já que a maior exposição a fatores iatrogênicos e à possibilidade de associação de múltiplos indicadores de risco acabam por tornar os recém-nascidos, com indicadores de riscos, mais propensos a apresentarem desvios do desenvolvimento da audição (4-6).

Em estudo retrospectivo (7), realizado em uma população de neonatos, o baixo peso/pequeno para idade gestacional (PIG) foi apontado como sendo um dos principais indicadores de risco para perda auditiva no grupo de recém-nascidos pré-termo, seguida de uso de ototóxico e ventilação mecânica. Em relação aos recém-nascidos a termo, os autores apontaram infecção congênita, antecedente familiar, o uso de ototóxico e o baixo peso/pequeno para idade gestacional (PIG) como os indicadores de risco mais frequentes para as alterações auditivas. Em outro estudo (8), ao analisarem as diferentes condições perinatais associadas à presença de distúrbios clínicos da comunicação, o baixo peso ao nascimento foi identificado pelas mães das crianças estudadas como a variável de maior ocorrência.

Assim também, as anomalias craniofaciais, tal como a fissura labiopalatina, consta da lista dos indicadores de risco para a audição (9), e frequentemente são acompanhadas de manifestações clínicas associadas e distantes da cavidade oral (10). A deficiência auditiva é dos problemas bastante observados em crianças com este tipo de malformação (11).

As fissuras labiopalatinas são malformações, que dentre as anomalias craniofaciais, são as mais prevalentes, destacando-se pelo número de alterações e pela complexidade de seus efeitos estéticos e funcionais. A literatura relata que a audição dessa população parece estar associada a efeitos em todos os níveis do sistema auditivo, à orelha média, por meio de perda auditiva condutiva; à cóclea, pela perda auditiva sensorioneural de frequência alta; ao tronco encefálico e vias auditivas centrais, pelo transtorno de processamento auditivo (12).

Considerando que tanto a população nascida com baixo peso ao nascimento, assim como com uma malformação craniofacial figuram entre os indicadores de risco para a deficiência auditiva, julgou-se necessário realizar um estudo retrospectivo comparativo dos achados audiométricos e timpanométricos de crianças nascidas com peso abaixo de $2500 \mathrm{~g}$ e portadoras de fissura labiopalatina, visando verificar se a presença conjunta desses dois indicadores determina o aparecimento de um comprometimento auditivo maior. Além de que, a literatura científica enfocando esta associação tem se mostrado carente. Assim, tendo conhecimento das causas determinantes da dificuldade auditiva apresentada por essa população de nossa vivência clínica, será possível fornecer orientação, bem como elaborar intervenções que reduzam e previnam a ocorrência das mesmas.

Os objetivos deste estudo foram verificar e comparar a audição de crianças com fissura labiopalatina com e sem baixo peso ao nascimento na avaliação audiológica convencional.

\section{MÉTODO}

Após aprovação pelo Comitê de Ética em Pesquisa em Seres Humano (Processo No 096/2009), foi realizado um estudo retrospectivo de dados audiológicos de 73 crianças de sete a 12 anos de idade, constantes em seus prontuários, escolhidos aleatoriamente, cuja avaliação audiológica foi realizada de julho de 2004 a junho de 2007, por uma mesma fonoaudióloga, em um hospital público especializado no atendimento de anomalias craniofaciais. Todas as crianças incluídas neste estudo não apresentavam qualquer síndrome genética associada. Esta pesquisa foi realizada no ano de 2009.

Dos prontuários foram verificados os dados referentes ao gênero, ao peso ao nascimento, à presença/ausência de fissura labiopalatina e, à avaliação audiológica convencional (audiometria e imitanciometria).

A análise do peso ao nascimento, assim como da presença e ausência de fissura labiopalatina tiveram o objetivo de selecionar a casuística desse estudo retrospectivo. Da compilação desta análise três grupos foram constituídos: 
- Grupo 1 (G1): 23 crianças com fissura labiopalatina e com baixo peso ao nascimento (faixa de 1750 a 2490grs, peso médio de 2218grs).

- Grupo 2 (G2): 25 crianças com fissura labiopalatina e sem baixo peso ao nascimento (faixa de 2600 a 3850grs, peso médio de 3160grs).

- Grupo 3 (G3): 25 crianças sem fissura labiopalatina e sem baixo peso ao nascimento (faixa de 2500 a 4100grs, peso médio de 3328grs).

A Tabela 1 apresenta a distribuição entre os grupos, segundo a idade e gênero.

Salienta-se que nem todo bebê com baixo peso ao nascer é um bebê pré-termo, e que nem todo bebê prétermo apresenta baixo peso.

Assim neste estudo, o termo "baixo peso ao nascer" será utilizado independentemente da idade gestacional.

A classificação dos pesos ao nascimento seguiu Fernández-SANABria (2).

Seguindo o objetivo proposto, na audiometria foram verificadas a presença e ausência de perda auditiva. Nos casos que apresentaram presença, foram classificados o tipo e o grau da perda auditiva.

O tipo de perda auditiva foi classificado em perda auditiva condutiva; perda auditiva sensorioneural; perda auditiva mista (13).

Foi considerada audição normal quando os limiares audiométricos se encontravam de zero a $20 \mathrm{~dB}$. A presença de perda auditiva foi estabelecida quando em qualquer uma das frequências testadas, de $250 \mathrm{~Hz}$ a $8 \mathrm{kHz}$, o limiar foi igual ou superior a $21 \mathrm{~dB}$. Desta forma, as perdas auditivas foram classificadas, quanto ao grau: leve (entre 21 e $40 \mathrm{~dB}$ ); moderado (entre 41 e $70 \mathrm{~dB}$ ); severo (entre 71 e $90 \mathrm{~dB}$ ) e, profundo (acima de $91 \mathrm{~dB}$ ) (14).

As audiometrias foram realizadas com a utilização do audiômetro Madsen, modelo Midimate 622, fones supraaurais TDH 39, na determinação dos limiares tonais, bem como no índice percentual de reconhecimento de fala de cada orelha.

Quanto a imitanciometria, foram verificados os tipos de curvas timpanométricas, assim como a presença e ausência de reflexos acústicos.

Nas timpanometrias realizadas foi utilizado o Imitanciômetro Grason Stadler Middle Ear Analyzer versão 2. A frequência do tom da sonda de imitância foi de $226 \mathrm{~Hz}$ (convencional). As medidas timpanométricas fo-
Tabela I . Distribuição dos grupos amostrados de acordo com a idade e gênero.

\begin{tabular}{|c|c|c|c|c|c|c|c|}
\hline \multirow[t]{2}{*}{ Idade (anos) } & \multicolumn{2}{|c|}{ Gl } & \multicolumn{2}{|c|}{$\mathrm{G} 2$} & \multicolumn{2}{|c|}{ G3 } & \multirow[t]{2}{*}{ Total } \\
\hline & $M$ & $F$ & $M$ & F & $M$ & $\mathrm{~F}$ & \\
\hline 7 & 0 & 2 & | & 4 & 4 & 3 & 14 \\
\hline 8 & 2 & 1 & 3 & 2 & | & | & 10 \\
\hline 9 & 5 & 0 & 2 & 3 & | & 3 & 14 \\
\hline 10 & 7 & 1 & 0 & 5 & 5 & 2 & 20 \\
\hline 11 & 0 & 2 & 3 & 0 & 2 & 0 & 7 \\
\hline 12 & 3 & 0 & 2 & 0 & 0 & 3 & 8 \\
\hline Total & 17 & 6 & 11 & 14 & 13 & 12 & 73 \\
\hline
\end{tabular}

Legenda: $\mathrm{M}=$ masculino; $\mathrm{F}=$ feminino.

ram realizadas automaticamente pelo equipamento, na velocidade de 50 decapascals por segundo $(\mathrm{daPa} / \mathrm{s})$. O tipo de curva timpanométrica obtido seguiu a classificação proposta (15). As curvas timpanométricas foram classificadas em normal e anormal. Normal quando foi obtida a curva tipo A, e anormal para os demais tipos encontrados (B, C, As e Ad).

Uma análise comparativa para cada grupo (gênero e idade) e entre os grupos foi realizada.

A análise estatística foi realizada por meio do teste qui-quadrado para a comparação do tipo da curva timpanométrica, presença e tipo de perda auditiva. Para o grau da perda auditiva utilizou-se o teste de KruskallWallis e Dunn. A comparação entre os lados, direito e esquerdo, foi realizada por meio do teste de McNemar para o tipo de curva, presença e tipo de perda auditiva; e teste de Wilcoxon para o grau da perda auditiva. $\mathrm{Na}$ comparação do peso e idade foi utilizado o teste Anova. Em todos os testes foi adotado nível de significância de 5\% $(\mathrm{p}<0,05)$.

\section{RESULTADOS}

A análise estatística não demonstrou diferença estatística significativa entre os grupos na comparação da idade $(p=0,368)$, do gênero $(p=0,099)$, e do peso entre G2 e G3 $(\mathrm{p}=0,342)$.

Quanto ao gênero e entre os lados, não houve diferença estatística significativa na orelha direita, em relação ao tipo da curva timpanométrica $(\mathrm{p}=0,733)$, presença de perda auditiva ( $p=0,915)$, tipo de perda auditiva $(\mathrm{p}=0,087)$ e grau da perda auditiva $(\mathrm{p}=0,855)$, assim como para a orelha esquerda quanto ao tipo da curva timpanométrica $(p=0,700)$, presença de perda auditiva $(p=0,952)$, tipo de perda auditiva $(p=0,696)$ e grau $d a$ perda auditiva $(p=0,969)$. 
Tabela 2. Comparação dos grupos amostrados.

\begin{tabular}{|c|c|c|c|c|c|c|c|c|c|c|}
\hline Variável & OD & GI & $\begin{array}{l}\mathrm{OE} \\
\mathrm{G} 2 \\
\end{array}$ & G3 & $\begin{array}{c}\text { Total } \\
\mathrm{p}\end{array}$ & GI & $\mathrm{G} 2$ & G3 & P & \\
\hline Perdaauditiva & $\begin{array}{l}\text { Sim } \\
\text { Não }\end{array}$ & $\begin{array}{l}\text { II (48\%) } \\
12(52 \%)\end{array}$ & $\begin{array}{l}8(32 \%) \\
17(68 \%)\end{array}$ & $\begin{array}{c}2(8 \%) \\
23(92 \%)\end{array}$ & $\begin{array}{l}0,009 * \\
G 1 \neq G 3 \\
G 2 \neq G 3\end{array}$ & $14(6 \mid \%)$ & $16(64 \%)^{2}$ & 5 ( $100 \%)$ & $0,002 *$ & 107 \\
\hline $\begin{array}{l}\text { Tipo de perda } \\
\text { auditiva }\end{array}$ & $\begin{array}{l}\text { Condutiva } \\
\text { Sensorioneural } \\
\text { Mista }\end{array}$ & $\begin{array}{l}10(9 \mid \%) \\
1(9 \%) \\
0(0 \%)\end{array}$ & $\begin{array}{l}6(75 \%) \\
\text { I (13\%) } \\
\text { I (13\%) }\end{array}$ & $\begin{array}{l}\text { I (50\%) } \\
\text { I (50\%) } \\
0(0 \%)\end{array}$ & $\begin{array}{l}0,049 * \\
G \mid \neq G 3\end{array}$ & $\begin{array}{l}8(89 \%) \\
1(11 \%) \\
0(0 \%)\end{array}$ & $\begin{array}{l}7(78 \%) \\
2(22 \%) \\
0(0 \%)\end{array}$ & $\begin{array}{l}0(0 \%) \\
0(0 \%) \\
0(0 \%)\end{array}$ & $\begin{array}{l}0,01 \mid * * \\
G \mid \neq G 3 \\
G 2 \neq G 3\end{array}$ & $\begin{array}{c}32 \\
6 \\
1\end{array}$ \\
\hline $\begin{array}{l}\text { Grau da perda } \\
\text { auditiva }\end{array}$ & $\begin{array}{l}\text { Leve } \\
\text { Moderada }\end{array}$ & $\begin{array}{l}9(82 \%) \\
2(18 \%)\end{array}$ & $\begin{array}{c}8(100 \%) \\
0(0 \%)\end{array}$ & $\begin{array}{c}2(100 \%) \\
0(0 \%)\end{array}$ & $\begin{array}{l}0,007 * \\
G \mid \neq G 3\end{array}$ & $\begin{array}{l}7(78 \%) \\
2(22 \%)\end{array}$ & $\begin{array}{c}9(100 \%) \\
0(0 \%)\end{array}$ & $\begin{array}{l}0(0 \%) \\
0(0 \%)\end{array}$ & $\begin{array}{l}0,002 * \\
G \mid \neq G 3\end{array}$ & $\begin{array}{c}35 \\
4\end{array}$ \\
\hline
\end{tabular}

* estatisticamente significante $(\mathrm{p}<0,05)$

Tabela 3. Distribuição da ocorrência das curvas timpanométricas dos grupos amostrados.

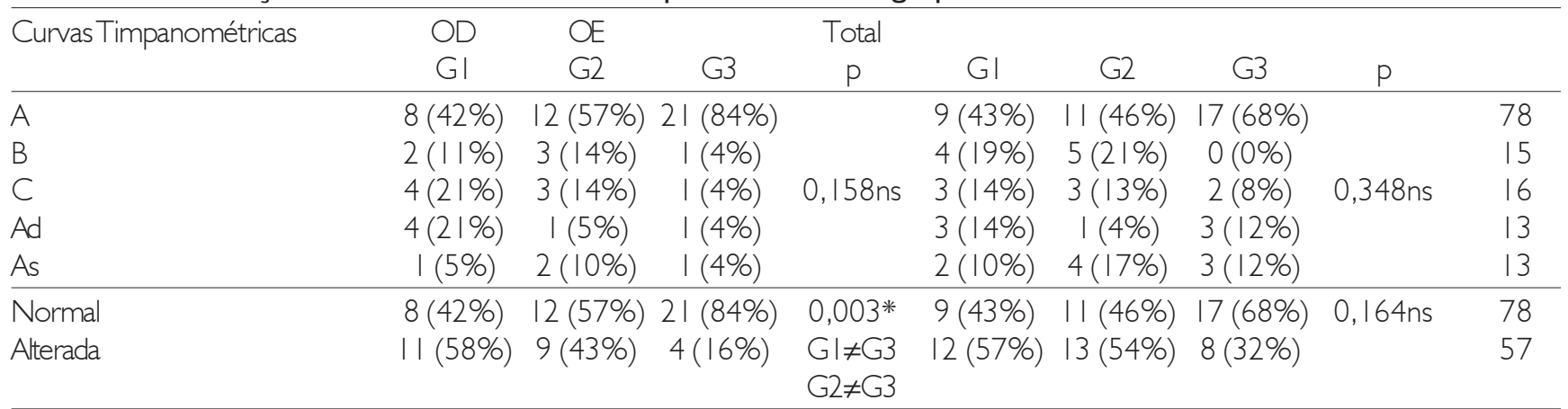

* estatisticamente significante $(\mathrm{p}<0,05)$

ns - não significante

O resultado da análise comparativa no que se refere à presença, ao tipo e ao grau de perda auditiva nos três grupos amostrados, está mostrado na Tabela 2.

Da população total amostrada 107 orelhas apresentaram limiares audiométricos dentro dos padrões de normalidade, sendo 26 pertencentes ao G1, 33 ao G2 e 48 ao G3.

A Tabela 3 mostra a distribuição da ocorrência e comparação entre os três grupos das curvas timpanométricas.

Os reflexos acústicos foram compatíveis com o resultado da avaliação audiológica.

\section{DISCUSSÃO}

No que se refere à presença de perda auditiva, pôde-se visualizar (Tabela 2 ) resultados sem significância estatística para os grupos de crianças com fissura labiopalatina, tanto para as nascidas com baixo peso quanto para as nascidas sem, o mesmo não ocorrendo em relação às não portadoras da malformação craniofacial e sem baixo peso ao nascimento, cuja perda de audição foi encontrada, somente, em duas orelhas. Este achado leva-se a pensar que a fissura é um componente muito mais importante para a audição do que o baixo peso. Estudos $(6,7,9,16)$ frequentemente relacionam problemas auditivos à presença de fissura labiopalatina e/ou baixo peso ao nascimento. No entanto, as alterações encontradas em nascidos de baixo peso podem ser individuais e dependentes de quão baixo era o peso da criança (17). Assim o resultado obtido neste estudo, sem significância pode estar relacionado ao peso das crianças de baixo peso, que se mostraram próximos aos das crianças dos outros dois grupos estudados e sem significância estatística. Literaturas mais atuais têm explorado nascidos com extremo e muito baixos peso, principalmente os menores de 1000grs, uma vez que eles são comumente expostos a outros fatores de riscos para a audição, tais como drogas ototóxicas, hipóxia e hiperbilirubinemia, que podem conduzir ao aparecimento de uma perda auditiva sensorioneural (18, 19, 20), além de perda condutiva (21).

A audição dentro dos padrões de normalidade, constatada em $65 \%$ da amostra de pacientes com fissura labiopalatina, está em consonância com estudo (22), no qual foi encontrada mais da metade das crianças avaliadas 
(63\%) com audição normal. Pesquisadores (23) também identificaram ausência de perda auditiva em 77\% das crianças com fissura labiopalatina não sindrômicas em trabalho realizado. Estudo (24) observou audição normal e ausência de anormalidades otoscópicas, mais frequentemente em crianças que foram submetidas à cirurgia do lábio pela técnica de Millard e do palato por meio da palatoplastia Langenbeck com veloplastia intravelar. No presente estudo não foi levado em conta à idade, nem a técnica cirúrgica do lábio e palato que as crianças com fissura labiopalatina foram submetidas.

Em relação ao tipo de perda auditiva, pôde-se verificar que a perda auditiva condutiva foi a de maior ocorrência para os grupos de crianças com fissura labiopalatina com (G1) e sem baixo peso ao nascimento (G2). Perda auditiva condutiva de grau leve a moderada é o achado audiométrico de maior ocorrência na população com fissura labiopalatina (25-27), concordante com o presente estudo, uma vez que estes graus foram encontrados no G1 e G2 em proporção maior significativamente que outros tipos.

No que se refere a imitanciometria todos os tipos de curvas timpanométricas estiveram presentes para os três grupos amostrados, com 58\% (78 orelhas) de timpanogramas A classificados como normal, uma vez que este tipo revela funcionamento do sistema tímpano-ossicular normal. Estudos $(24,28,29)$ corroboram com este achado.

Uma porcentagem de 42 (57 orelhas) foi classificada como curva timpanométrica anormal, sendo observado um comportamento similar entre os grupos de crianças com fissura labiopalatina (G1 e G2) e uma menor ocorrência na população sem este tipo de anomalia craniofacial.

Entre as curvas timpanométricas anormais uma distribuição quase que homogênea, sem significância estatística, foi observada entre os grupos amostrados.

A curva tipo $\mathrm{C}$, a mais frequente entre as curvas timpanométricas anormais, neste estudo, esteve presente em 7 orelhas do G1, 6 do G2 e em 3 do G3, é demonstrativa de uma alta pressão negativa na orelha média, refletindo alterações da tuba auditiva (15), podendo ainda, estar associada com função normal de orelha média, assim como com presença de fluido (30), sugerindo uma transição entre essas duas condições (31).

O tipo B, apesar da ausência de significância estatística, foi mais prevalente na população com fissura labiopalatina (G1 e G2). Este tipo tem como causa mais comum a redução da mobilidade de membrana timpânica secundária a o fluido de orelha média, assim como a rigidez do tímpano (cicatrizes), presença de formação de tecido conjuntivo denso ao redor dos ossículos (timpanosclerose), colesteatoma ou tumor da orelha média (32). Obtidas em menor ocorrência, neste estudo, as curvas Ad (9,5\%) e As (9,5\%) demonstram, respectivamente, flacidez do sistema tímpano-ossicular, devido a decorrentes casos de otites médias e/ou disjunção da cadeia ossicular (33) e, estado de rigidez (15).

Ao estudar a audição de 44 crianças na faixa etária de 8 a 14 anos do sexo masculino e feminino, portadoras de fissura labiopalatina e/ou palatina não-sindrômica, pesquisadores (18), encontraram curva timpanométrica tipo C $(21,2 \%)$ como a de maior ocorrência, seguida pela curva tipo B (7,1\%), sendo a curva tipo Ad (3,5\%) a menos prevalente.

\section{CONCLUSÃO}

Este estudo permitiu observar um maior comprometimento da audição, evidenciado pela perda auditiva condutiva de grau leve a moderada nas crianças com fissura labiopalatina, independente da presença de baixo peso ao nascimento, quando comparada as sem este tipo de malformação craniofacial.

\section{REFERÊNCIAS BIBLIOGRÁFICAS}

1. Tiensoli LO, Goulart LMHF, Resende LM, Colosimo EA. Triagem auditiva em hospital público de Belo Horizonte, Minas Gerais, Brasil: deficiência auditiva e seus fatores de risco em neonatos e lactentes. Cad Saúde Pública. 2007, 23(6):431-41.

2. Fernández-Sanabria, RV. Valor de Hemoglobina em la gestante y su relación com el parto pretérmino y peso del recién nacido en pacientes atendidas en el Hospital Santa Rosa durante el periodo Abril 2001- Octubre 2001. [Dissertação]. Lima(Peru): Universidad del Peru-Facultad de Medicina. 2002.

3. Vieira EP, Miranda EC, Azevedo MF, Garcia MV. Ocorrência dos indicadores de risco para a deficiência auditiva infantil no decorrer de quatro anos em um programa de triagem auditiva neonatal de um hospital público. Rev Soc Bras Fonoaudiol. 2007, 12(3):214-20.

4. Oliveira LN, Lima MCMP, Gonçalves VMG. Acompanhamento de lactentes com baixo peso ao nascimento: aquisição de linguagem. Arq Neuropsiquiatr. 2003, 61(3-B):802-7.

5. Azevedo MF, Vieira RM, Vilanova LCP. Desenvolvimento auditivo de crianças normais e de alto risco. São Paulo: Plexus; 2001. 
6. Oliveira ES. Triagem auditiva em recém-nascidos prematuros no município de Itapetininga, São Paulo [tese]. São Paulo: Faculdade de Saúde Pública da Universidade de São Paulo, Departamento de Saúde Materno-Infantil; 2005.

7. Pereira PKS, Martins AS, Vieira MR, Azevedo MF. Programa de triagem auditiva neonatal: associação entre perda auditiva e fatores de risco. Pró-Fono. 2007, 19(3):267-78.

8. Peñazola-López YR, Castillo-Maya G, García-Pedroza F, Sánchez-LópezH.Hipoacusia-sordera asociada a condiciones perinatales adversas según registro en unidad especializada de la Ciudad de México. Análisis em función del peso al nacimiento. Acta Otorrinolaringol Esp. 2004, 55:252-9.

9. Joint Committee on Infant Hearing. Year 2007. Position Statement: Principles and Guidelines for Early Hearing Detection and Intervention Programs. Pediatrics. 2007, 120 (4):898-921.

10. Silva AA, Maudonnet OAQ. Complicações otológicas em crianças fissuradas. Rev Bras Otorrinolaringol. 1990, 56(4):151-4.

11. Mello J, Kumar S. Audiological findings in cleft palate patients attending speech camp. Indian J Med Res. 2007, 125:777-82.

12. Feniman MR. Saúde auditiva do indivíduo com anomalias craniofaciais: fissura labiopalatina. In: Jesus, MSV, Di Ninno CQMS (org). Fissura labiopalatina: fundamentos para a prática fonoaudiológica. São Paulo: Roca; 2009. p. 179-90.

13. Russo ICP, Santos TMM. Interpretação dos resultados da avaliação audiológica. In: Russo ICP, Santos TMM. A prática da audiologia clínica. São Paulo: Cortez; 1993. p.191-212.

14. BIAP recommendation $\mathrm{n}^{\circ} 02 / 1$ bis. Audiometric classification of hearing impairments. Disponível em: www.biap.org. Acesso em: 26 de maio de 2011.

15. Jeger, J. Clinical experience with impedance audiometry. Arch Otolaryng. 1970, 92:311-24.

16. Lichtig I, Monteiro SRG, Couto MIV, Haro FMB, Campos MSC, Vaz FAC, et al. Avaliação do comportamento auditivo e neuropsicomotor em lactentes de baixo peso ao nascimento. Rev Ass Med Brasil. 2001, 47(1):52-8.

17. Caçola P, Tatiana Godoy Bobbio TG. Baixo peso ao nascer e alterações no desenvolvimento motora realidade atual. Rev Paul Pediatr. 2010, 28(1):70-6.

18. Rugolo LMSS. Peso de nascimento: motivo de preocupação em curto e longo prazo J Pediatr. 2005, 81(5):359-60.

19. Fanaroff JM, Wilson-Costello DE, Newman NS, Montpetite MM, Fanaroff AA. Treated Hypotension Is Associated With Neonatal Morbidity and Hearing Loss in Extremely Low Birth Weight Infants. Pediatrics. 2006, 117(4):1131-5.

20. Cristobal R, Oghalai JS. Hearing loss in chilfren with very low birth weight: current review of epidemiology and pathophysiology. Arch Dis Child Fetal Neonatal Ed. 2008, 93:462-8.

21. Roth DA-E, Hildesheime M, Maayan-Metzger A, Muchnik C, Hamburger A, Mazkeret R, Kuint J. Low prevalence of hearing impairment among very low birth weight infants as detected by universal neonatal hearing screening. Arch Dis Child Fetal Neonatal Ed. 2006, 91:257-62.

22. Tunçbilek G, Özgür F, Belgin E. Audiologic and tympanometric findings in children with cleft lip and palate. Cleft Palate Craniofac J. 2003, 40(3):304-9.

23. Amaral MIR, Martins JE, Santos MFC. Estudo da audição em crianças com fissura labiopalatina não-sindrômica. Braz. J otorhinolaryngol. 2010, 76(2):164-71.

24. Antonelli P, Jorge J, Feniman M, Piazentin-Penna S, DutkaSouza J, Seagle, et al 2010. Otologic and audiologic outcomes with the Furlow and Von Langenbeck with intravelar veloplasty palatoplastics in unilateral cleft lip and palate. Disponível em: htth://www.cpcjournal.org/toc/cpcj/0/0 em 23.03.2011.

25. Paliobei V, Psifidis A, Anagnostopoulos D. Hearing and speech assessment of cleft palate patients after palatal closure. Long-term results. Int J Pediatr Otorhinolaryngol. 2005, 69(10):1373-81.

26. Ramana YV, Nanda V, Biswas G, Chittoria R, Ghosh S, Sharma RK. Audiological profile in older children and adolescents with unrepaired cleft palate. Cleft Palate Craniofac J. 2005, 42(5):570-3.

27. Souza D, Di Ninno CQMS, Borges GP, Silva TM, Miranda ES. Perfil audiológico de indivíduos operados de fissura de palato no hospital da baleia de Belo Horizonte. Acta ORL. 2006, 24(3):170-3.

28. Namyslowski G, Kubik P. 226Hz and high frequency tympanometry in children with cleft lip and/or palate. Otolaryngol Pol. 1996, 50(1):73-80.

29. Feniman MR, Souza AG, Jorge JC, Lauris JRP. Achados 
otoscópicos e timpanométricos em lactentes com fissura labiopalatina. Rev Bras Otorrinolaringol. 2008, 74(2): 24852.

30. Harris PK, Hutchinson KM, Moravec J. The use of tympanometry and pneumatic otoscopy for predicting middle ear disease. Am J Audiol. 2005, 14:3-13.

31. Combs JT. The diagnosis of otitis media: new techniques. Pediatr Infect Dis J. 1994, 13:1039-46.
32. Fowler CG, Shanks JE. Tympanometry. In: KatzJ, Burkard RF, Medwetsky L. Handbook of clinical audiology. 5 th ed. Philadelphia: Lippincott Williams \& Wilkins, 2002:175204.31. Pensak M. Cholesteatoma. In: Tami TA. Otolaryngology: a case study approach. New York: Thieme, 1998:17-20.

33. Carvallo MMR. Procedimentos em Audiologia. Em: Fonoaudiologia: Informação para Formação. $1^{a}$ edição. São Paulo: Ed Guanabara Koogan; 2003. 